\title{
Pulpotomy of Primary Molar Teeth with Biodentine - A Case Report
}

\author{
Sheikh MAH ${ }^{\mathrm{a}}$, Khanum $\mathrm{S}^{\mathrm{b}}$, Shaikh AK ${ }^{\mathrm{c}}, \mathrm{Nisa} \mathrm{SS}^{\mathrm{d}}$
}

\begin{abstract}
A 7-year-old boy was referred to the department of conservative dentistry and endodontics, Bangabandhu Sheikh Mujib Medial University (BSMMU) with pain on his left lower jaw. After clinical and radiological examination, it was diagnosed as a case of reversible pulpitis. Biodentine pulpotomy was performed followed by permanent restoration with glass-ionomer. The presented case was done to evaluate the efficacy of Biodentine as pulpotomy medicament in primary molar teeth, on follow-up it was found to be successful.
\end{abstract}

Key Words: Biodentine, Pulpotomy

(BIRDEM Med J 2017; 7(3): 238-241)

\section{Introduction}

Although there have been many advances in the prevention of dental caries and the understanding of the importance of preserving natural teeth has increased, many teeth are still lost at an early age. Preservation of primary teeth before the eruption of permanent teeth is desirable since they help to determine the shape of dental arches, maintain the space between teeth, prevent detrimental tongue and speech habits, preserve aesthetics and maintain chewing function. Hence, teeth with caries should ideally be restored rather than be extracted. ${ }^{1}$

When the carious process exposes the pulp, the tissue close to the lesion becomes inflamed. ${ }^{2}$ Pulpotomy is considered as one of the most widely accepted clinical procedure for preserving primary teeth affected by

Author information

a. Dr. (Major) Md Abdul Hannan Sheikh, BDS, MCPS, D. Conservative, FCPS. Classified Specialist in Conservative Dentistry \& Endodontist, CMH, Savar Cantt.

b. Dr. (Major) Shakila Khanum, MBBS, MCPS, FCPS. Classified Specialist in Gynae \& Obs, CMH, Savar Cantt.

c. Dr. Abdul Kader Sheikh, MBBS, FCPS, MD (Neurology) Associate Prof, Dept of Neurology, BSMMU, Dhaka

d. Dr. (Capt) Shamsia Sultana Nisa, BDS. Dental Surgeon, CMH, Savar Cantt.

Address of Correspondence: Dr. (Major) Md Abdul Hannan Sheikh, BDS, MCPS, D. Conservative, FCPS. Classified Specialist in Conservative Dentistry \& Endodontist, CMH Savar Cantt. E-mail: drhannan65@gmail.com

Received: February 22, 2017

Accepted: July 31, 2017 inflammation of the coronal pulp caused by caries with no involvement of the radicular pulp. Pulpotomy is a vital pulp therapy in which a portion of vital coronal pulp tissue is removed surgically and the remaining radicular dental pulp is covered with a suitable material that protects the pulp from further injury and permits and promotes healing. ${ }^{3}$ Medicaments applied into the radicular tissue following the removal of coronal pulp are formocresol, ferric sulphate, gluteraldehyde, calcium hydroxide, mineral trioxide aggregate (MTA), Portland cement etc.

Biodentine is a new class of dental material which is also considered as effective in conventional pulp therapy. It is calcium silicon cement based on "Active Biosilicate Technology" and composed of powder in capsule and liquid in pipette. The powder portion consist of tri-calcium silicate as main core material, di-calcium silicate as second core material, calcium carbonate and oxide as fillers, iron oxides as shade and zirconium oxide as radio-pacifier. The liquid portion consists of calcium chloride as accelerator and hydrosoluable polymer as water reducing agents. ${ }^{4}$

The powder is mixed with the liquid in a capsule in the triturator for 30 seconds. Once mixed, Biodentine sets in approximately 12 minutes. The consistency of Biodentine is similar to that of phosphate cement. Biodentine can be used on both crowns and roots. Its crown application include pulp protection, temporary closure, deep caries management, cervical filling, direct and indirect pulp capping and pulpotomy. Its use in roots includes managing perforations of root canals or the 
pulp floor, internal and external root resorption, apexification and retrograde root canal obturation. ${ }^{5}$

In this case report, pulpotomy with Biodentine was performed to maintain the pulp vitality of a primary molar tooth.

\section{Case Report}

A 7-year-old boy was referred to the department of conservative dentistry and endodontics, BSMMU with mild pain and food accumulation during taking food on his lower left jaw for few days. The pain resolves after removal of the food. On clinical examination, the lower left second primary molar had deep carious lesion (Fig-1). The tooth was sensitive to cold. No visible swelling and sinus tract was found. Radiological examination revealed that the tooth had no periapical pathology (Fig.-2). His medical history was non significant. A diagnosis of reversible pulpitis due to caries was made and pulpotomy with biodentine was planned.

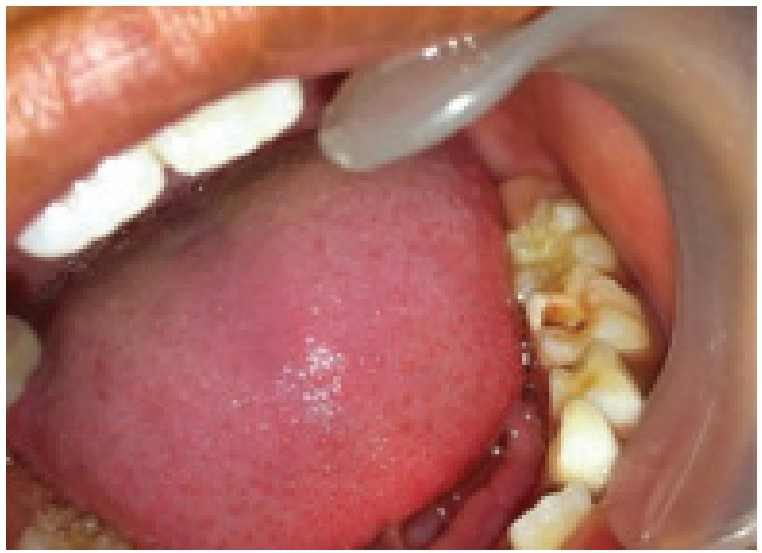

Figure 1. Intraoral photograph

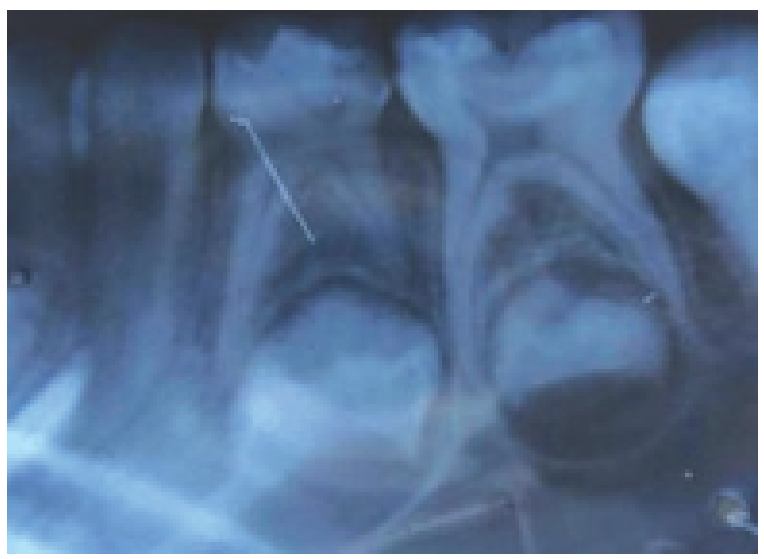

Figure 2. Initial radiograph
Treatment procedure was explained to the patient and his parents and informed consent was obtained. Disinfection of the operative field and proper sterilization of the instruments was ensured. Local anaesthesia was given. After proper isolation, surrounding caries was removed by using no. 4 round burs in a high speed hand piece using copious water spray. Then, roof of pulp chamber was removed similarly. Coronal pulp from pulp chamber was removed by using sterile excavator until the orifices of canal are seen. Finally, the pulp chamber was rinsed with normal saline (Fig-3) and the orifices were covered with a small cotton pellet soaked in normal saline with pressure until bleeding is controlled. Biodentine Cement was mixed according to manufacturer instruction (Fig-4) and applied over the amputed pulp of the prepared cavity. Then, by using a sterile cotton pellet Cement was condensed properly (Fig-5). After setting of the Biodentine, the rest of the cavity was filled with Fuji1X Glassionomer cement. Bite was checked for any high spots \& two coats of varnish was applied over the Glass-

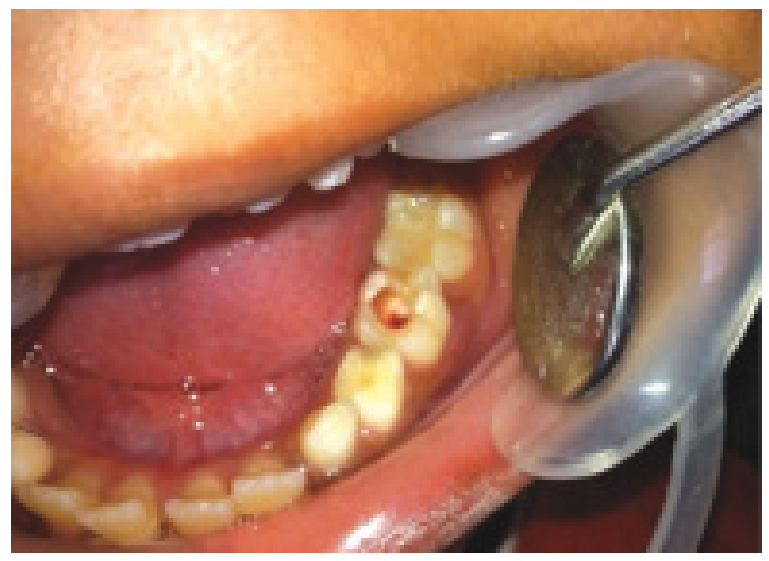

Figure 3. After coronal pulp tissue removal

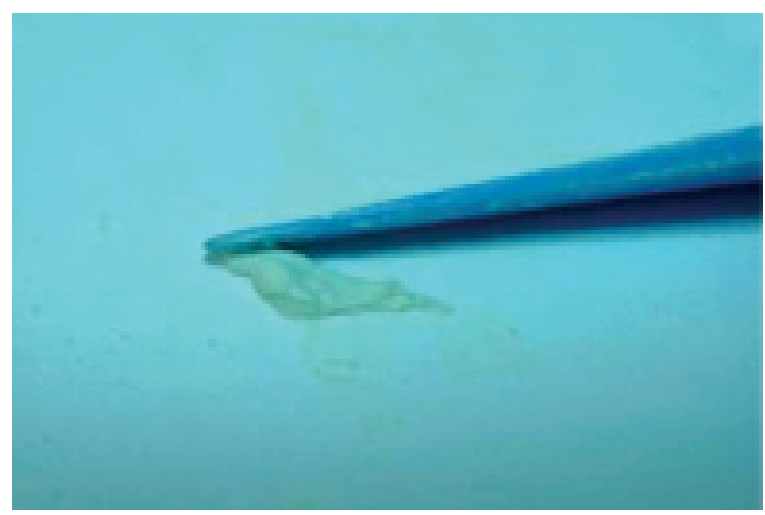

Figure 4. Mixing of biodentin 


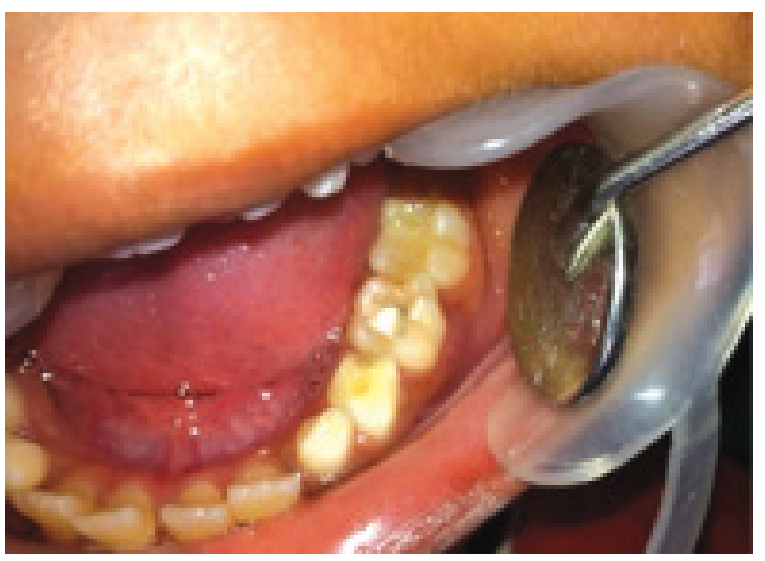

Figure 5. Placement of biodentin

ionomer. Post operative radiograph was taken. Patient was recalled after 3, $6 \& 12$ months. Follow up upto 12 months the tooth was functional with no signs \& symptoms both clinically \& radiographically (Fig-6,7,8).

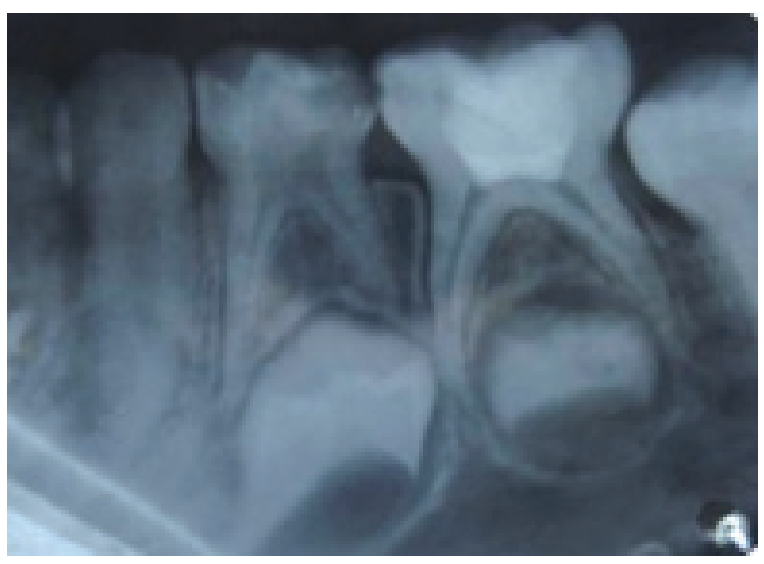

Figure 6. Follow up X-ray after 3 months

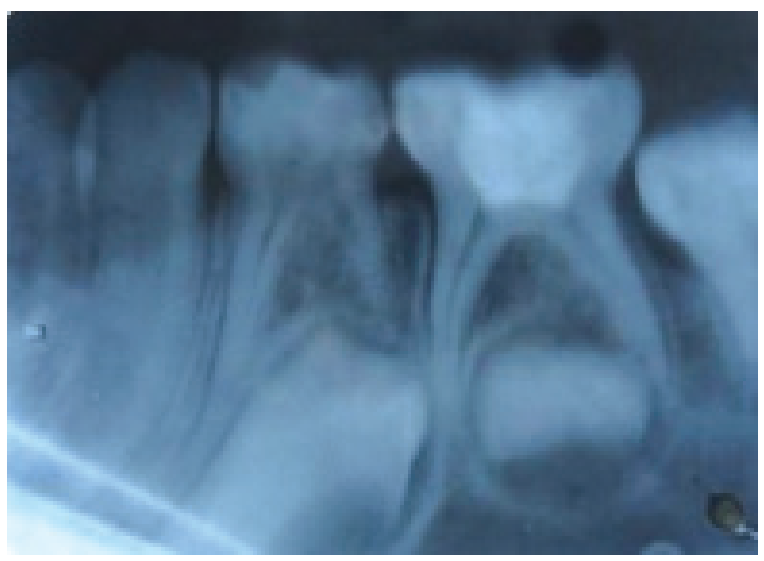

Figure 7. Follow up X-ray after 6 months

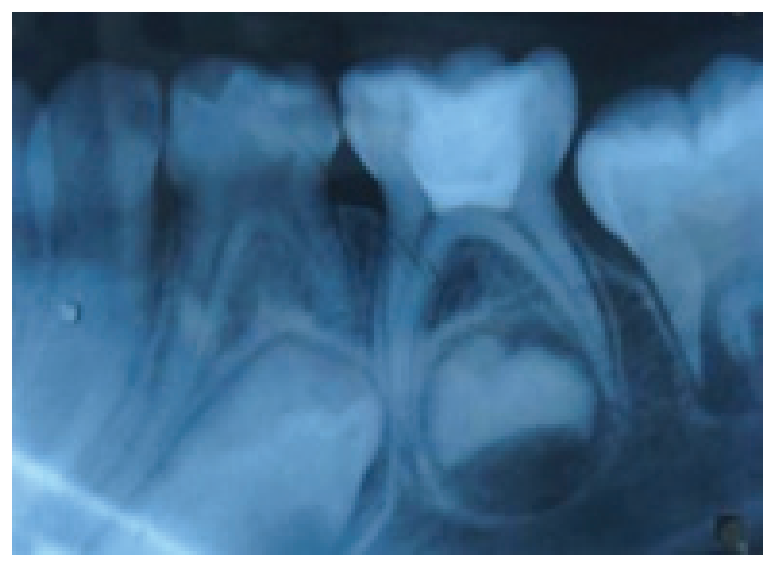

Figure 8. Follow up X-ray after 12 months

\section{Discussion}

The retention of pulpuly involved deciduous tooth in a healthy state until the of normal exfoliation remains to be one of the challenges for paedodontists. Pulpotomy is the common therapy for cariously exposed pulps in symptom-free primary molars, its aim being to preserve the radicular pulp to avoid pain and swelling, ultimately to retain the tooth.

Dilute formocresol is regarded as the 'gold standard' but its use as pulpotomy agent is still controversial because many previous studies have reported that formocresol has toxic mutagenic and carcinogenic risk in humans. These includes nasopharyngeal cancers in humans, limited evidence of nasal and paranasal sinuses cancers, and strong but not, sufficient evidence for leukemia, an increase in the prevalence of hypoplastic and or hypomineralization defects and prevalence in positional alteration of the succedaneous. Furthermore, necrosis and sloughing of the gingival tissue have been reported when comes in contact. ${ }^{6-9}$

The international agency for research cancer of the world health organization recently reclassified formaldehyde as a known human carcinogen. For decades calcium hydroxide has been the material of choice among the available pulpotomy materials. Many researchers agree that calcium hydroxide encourages the formation of dentinal bridge. ${ }^{10-11}$ Despite the wide use of calcium hydroxide, many studies showed that it has certain drawbacks such as it does not adhere to dentin and will dissolve over time. Moreover, the hard tissue bridge under calcium hydroxide has many imperfections and tunnel defects that may permit bacterial microleakage. ${ }^{12}$ 
To overcome this situation in the presented case Biodentine was used. Biodentine is a cement of same class as MTA. ${ }^{13}$ MTA has some disadvantage like its handling is somewhat difficult, the setting time is relatively long, the values for compressive and flexural strength are markedly lower than those for dentine and it is comparatively expensive. On the other hand Biodentine is mostly simpler to use, setting time is below that of MTA, it has a creamy stone like texture. Biodentine has physical properties similar to dentine. On the biological level, it is perfectly biocompatible and capable of inducing the apposition of reactionary dentin by stimulating odontoblast activity and reparative dentin, by induction of cell differentiation. It is an effective dentin substitute that can be used as a coronal restoration material but can also be placed in contact with the pulp. ${ }^{13}$ The potential applications of Biodentine on pulp therapy have already been explored by a number of investigators. Animal histological studies have showed that no sign of pulp cell damage could be identified after placing Biodentine into animal and human pulp as pulp capping material. Furthermore, Biodentine stimulates the expression of collagen, dentine sialoprotein and osteonectine involved in mineralization in cultured human pulp cells. ${ }^{4}$

The action mechanism of calcium silicate cement such as Biodentine involves the release of calcium hydroxide with a basic $\mathrm{pH}$ like calcium hydroxide with in addition, impervious dentin material interfaces as well as a dissolution resistance that does not involve any reintervention. ${ }^{13}$

Biodentine also promotes the precipitation of dentin bridge formation. The mechanism of hard tissue formation was also examined. Clinical evaluation by Lucile Goupy et al. reported that two cases of Biodentine cement showed effective when used as medicaments following cervical pulpotomy on a maxillary first deciduous molar and partial pulpotomy following pulp exposure during caries excavation on permanent molar. ${ }^{13}$

\section{Conclusion}

Biodentine is an interesting, very promising product, which with correct diagnosis can certainly contribute to a high degree to maintenance of the vitality of the dental pulp or to the retention of a tooth. More scientific studies on Biodentine are therefore absolutely necessary.

\section{References}

1. Barr ES, Flaitz CM, Hicks MJ. A retrospective radiographic evaluation of primary molar pulpectomies. Pediatric Dentistry, 1991;13:4-9.

2. Eidelman E, Holan G, Fucks AB. Mineral Trioxide Aggregate VS Formocresol. In pulpotornized primary molars a preliminary report. Pediatric Dentistry 2001;23:15-18.

3. Bakland. Endodontic consideration in dental trauma. In: Ingle JI, Bakland LK. eds. Endodontics. Toronto: BC Decker Inc, 2002; pp795-844.

4. Biodentine ${ }^{\mathrm{TM}}$ - Active Biosilicate Technology. Scientific File. Septodont, Paris 2010.

5. Dammaschke T. A new bioactive cement for pulp capping, Internatinal Dentsitry (African Edition) 2012;2(2): 64-68.

6. De Deus G, Ximenes R, Gurgel Filho E, Plotkowski M, Coutinho Filho T. Cytotoxicity of MTA and Portland cement on human ECV 304 endothelial cells. International Endodontic Journal 2005; 38(9):604-9.

7. Menezes R, Brarnante C M, Letra A, Carvalho V G G, Garcia R B. Histologic evaluation of pulpotomies in dog using two types of mineral trioxide aggregate and white Portland cements as wound dressings. Oral Surg Oral Med Oral Pathol Oral Radiol Endod 2004; 98: 376-79.

8. Schwartz RS, Mauger M, Clement DJ, Walker WA. Mineral trioxide aggregate: a new material for endodontics. J Am Dent Assoc 1999; 130: 967-75.

9. Tanomaru-Filho M, Tanomaru JMG, Barros DB, Watanabe E, Ito IY. In vitro antimicrobial activity of endodontic sealers, MTA-based cements and Portland cement. Journal of Oral Science 2007;49(1):41-5.

10. Kopel HM. Consideration for the direct pulp capping procedure in the primary teeth: a review of the literature. ASDC J Dent Child 1992;59:141-49.

11. HØrsted-Bindslev P, Vilkinis V, Sidlauskas A. 2203, Direct capping of human pulps with a dentine bonding system or with calcium hydroxide cement Oral Surg Oral Med Oral Pathol Pral Radiol Endo, 2003;96:591-600.

12. Cox CF, Suhay RK, Ostro E, Suzuki S, Suzuki SH. 1996, Tunnel defects in dentin bridges: their formation following direct pulp capping. Oper Dent. 1996;21:4-11.

13. Goupy L. Biodentine a novel substitute for use in pediatric dentistry. Septo dent Case Studies 2012;1:12-16. 